\title{
Application of a VUV Fourier transform spectrometer and synchrotron radiation source to measurements of. VI. The $\epsilon(0,0)$ band of NO
}

\author{
A. S-C. Cheung, A. L. Wong, D. H-Y. Lo, and K. W-S. Leung \\ Department of Chemistry, The University of Hong Kong, Hong Kong \\ K. Yoshino \\ Harvard-Smithsonian Center for Astrophysics, Cambridge, Massachusetts 02138 \\ A. P. Thorne and J. E. Murray \\ Blackett Laboratory, Imperial College, London, SW7-2BZ, United Kingdom \\ T. Imajo \\ Japan Women's University, Tokyo 112-8681, Japan \\ K. Ito and T. Matsui ${ }^{\mathrm{a})}$ \\ Photon Factory, KEK, Tsukuba, Ibaraki 305, Japan
}

(Received 28 March 2003; accepted 28 July 2003)

\begin{abstract}
The $\epsilon(0,0)\left(D^{2} \Sigma^{+}-X^{2} \Pi_{r}\right)$ band of NO has been recorded by using a vacuum ultraviolet Fourier transform spectrometer from Imperial College, London, with synchrotron radiation at the Photon Factory, KEK, Japan, as a continuum light source. Analysis of the $\epsilon(0,0)$ band provides accurate rotational line positions and term values as well as the photoabsorption cross sections. Molecular constants of the $v=0$ level of the $D^{2} \Sigma$ state have been determined as $T_{0}=53291.10$ $\pm 0.10 \mathrm{~cm}^{-1}, \quad B_{0}=1.99107 \pm 0.00005 \mathrm{~cm}^{-1}, \quad$ and $\quad D_{0}=(6.6 \pm 0.1) \times 10^{-6} \mathrm{~cm}^{-1}$. Accurate rotational line strengths have also been obtained and the sum of the line strengths for all rotational lines is determined as $2.18 \times 10^{-15} \mathrm{~cm}^{2} \mathrm{~cm}^{-1}$. The band oscillator strength of the $\epsilon(0,0)$ band is determined to be $(2.47 \pm 0.12) \times 10^{-3}$. (c) 2003 American Institute of Physics.
\end{abstract}

[DOI: $10.1063 / 1.1611171]$

\section{INTRODUCTION}

In the wavelength region 175-205 $\mathrm{nm}$ the penetration of solar radiation into the atmosphere is controlled by the absorption cross sections of the Schumann-Runge bands of $\mathrm{O}_{2}$. Part of the radiation transmitted is available to photopredissociate NO, which has a number of strong bands: namely $\gamma$ system $\left(A^{2} \Sigma^{+}-X^{2} \Pi\right), \beta$ system $\left(B^{2} \Pi-X^{2} \Pi\right), \delta$ system $\left(C^{2} \Pi-X^{2} \Pi\right)$; and $\epsilon$ system $\left(D^{2} \Sigma^{+}-X^{2} \Pi\right)$, with very narrow lines in the region $183-195 \mathrm{~nm}$. With recent advancement of new spectroscopic techniques, we thought it would be beneficial to record and analyze these NO systems with a resolution that is comparable to the Doppler width to obtain accurate line positions and, more importantly, the band oscillator strengths of these bands. Discussions of the necessity for high-resolution measurements of NO have been presented in previous papers of the series on NO bands. ${ }^{1-5}$ In these papers we describe how we combined high-resolution vacuum ultraviolet (VUV) Fourier transform spectrometry (FTS) with synchrotron radiation by taking the Imperial College (IC) VUV FT spectrometer to the synchrotron radiation source at the Photon Factory, KEK, Japan, where a suitable zero-dispersion two-grating predisperser is available on beamline 12-B. (The predisperser is necessary to limit the bandwidth to a few $\mathrm{nm}$ in order to achieve acceptable signalto-noise ratios $S / N$.) We have used this combination of fa-

\footnotetext{
${ }^{a)}$ Present address: National Institute of Advanced Industrial Science and Technology, Ibaraki 305-8565, Japan.
}

cilities to make ultrahigh resolution cross-section measurements of NO in the wavelength region 160-195 nm. The present paper in this series presents the spectroscopic analysis and the line and band oscillator strength of the $\epsilon(0,0)$ $\left(D^{2} \Sigma^{+}-X^{2} \Pi_{r}\right)$ band of NO.

The first measurements of the vacuum ultraviolet spectra of NO were made by Leifson, ${ }^{6}$ who photographed the absorption bands of the molecules in 1926 and noticed the three groups of band systems, now known to be the $\gamma, \beta$, and $\epsilon$ bands. An extensive review of early experimental work on the electronic spectrum of NO is given by Miescher and Huber. ${ }^{7}$ The $\epsilon$ bands were thought to be a continuation of the $\gamma$ bands. ${ }^{8,9}$ Ogawa ${ }^{10}$ recognized the distinct identity of the $\epsilon$ bands as well as the $\gamma$ bands from discontinuities observed in the rotational constants, $\Delta G$ values, and intensities. Herzberg et al. ${ }^{11}$ confirmed it by observing the $\gamma(4,0)$ band in the $\epsilon(0,0)$ band. Rotational analyses of the $\epsilon(0, v)$ bands in emission have been done, and molecular constants of the $D(0)$ level are available. ${ }^{10,12-14}$ Barrow and Miescher ${ }^{15}$ presented rotational analyses of the absorption bands of the $\epsilon$ system with $v^{\prime}=0-4$ and $\gamma$ system with $v^{\prime}=4-5$, but failed to present the entire list of the rotational assignments. They discussed the weak interaction between the $D(0) F_{2}$ [the upper level of the $\epsilon(0,0)$ band] and $B(8) F_{2}$ at $J=3.5$. Amiot and Verges ${ }^{16}$ observed the fine structure of the $D(0)-A(0)$ band in emission with a high-resolution Fourier transform spectrometer in the infrared region. They presented accurate molecular constants and term values measured from $A^{2} \Sigma^{+}$ 
$(v=0, J=0.5)$. Recently Braun et al. ${ }^{17}$ observed the $D^{2} \Sigma^{+}-C^{2} \Pi$ bands in emission with a high-resolution Fourier transform spectrometer in the infrared region. They combined their $E \rightarrow C$ transition with $E \rightarrow D, D \rightarrow A, C \rightarrow A,{ }^{16}$ and also $C \leftarrow X,{ }^{18}$ and they tabulated the term values of the $A, D, E$, and $C$ states for $v=0-2$.

The cross-section measurements of the $\epsilon(0,0)$ band by Bethke $^{19}$ were performed at low resolution, $0.04 \mathrm{~nm}$, in the presence of Ar. The addition of argon buffer gas ensured that the rotational lines were broadened beyond the instrumental resolution, allowing the true cross section to be presented. Cieslik $^{20}$ used low-resolution measurements of NO at pressure below 1 Torr with the curve of growth and the equivalent width to obtain the oscillator strengths of the $\epsilon(0,0)$ band. A similar method was employed by Callear and Pilling $^{21}$ with added Ar. Guest and Lee ${ }^{22}$ used a synchrotron source and resolution of $0.03 \mathrm{~nm}$ for the $\epsilon(0,0)$ band at very low pressure of NO gas, $2 \times 10^{-4}-7 \times 10^{-2}$ Torr. Chan et $a l .{ }^{23}$ used the high-resolution dipole $(e, e)$ technique, which is not sensitive to instrumental resolution. Luque and Crosley $^{24}$ also obtained the band oscillator strengths from the lifetime measurements after two-photon excitation.

In this paper, we report the analysis of the $\epsilon(0,0)$ band around $187.6 \mathrm{~nm}$ by using the VUV FTS with synchrotron radiation for the background source. Because of the capability of the combination of instruments, these are the first highresolution quantitative measurements of line positions and intensities of the rotational lines of the $\epsilon(0,0)$ band. The rotational term values of the $D^{2} \Sigma^{+}(v=0)$ levels have been evaluated and then used with a least-squares fitting program to obtain accurate molecular constants of this level. The determination of the band oscillator strengths of the band has been performed using line-by-line measurements, because the resolution of the present experiment is comparable to the Doppler widths.

\section{EXPERIMENT}

Details of the experimental procedures for recording high-resolution FT spectra of NO between 160 and $198 \mathrm{~nm}$ have been described in our earlier publications. ${ }^{1,2}$ Only a brief description of the experimental conditions with respect to the recording of the $\epsilon(0,0)$ band will be given here. An absorption cell with optical path length of $0.565 \mathrm{~cm}$ was used, which was filled with 0.300 Torr of NO at $295 \mathrm{~K}$. The column density of $\mathrm{NO}$ in this experiment was 5.55 $\times 10^{15} \mathrm{~mol} \mathrm{~cm}^{-2}$. A total of 272 coadded scans were taken with a resolution of $0.06 \mathrm{~cm}^{-1}$ corresponding to an approximate integration time of $12 \mathrm{~h}$. The signal-to-noise ratio in the continuum background was about 97 . The resonance line of $\mathrm{Hg} \mathrm{I}$ at $184.8 \mathrm{~nm}$ was used as the reference wave number in the previous papers. ${ }^{1,3}$ However, in this wavelength region we have no convenient absolute reference. From the calibration constant in the previous papers, we estimated the uncertainty in the absolute values as $0.1 \mathrm{~cm}^{-1}$. Relative uncertainties for the strong lines are better than $0.01 \mathrm{~cm}^{-1}$.
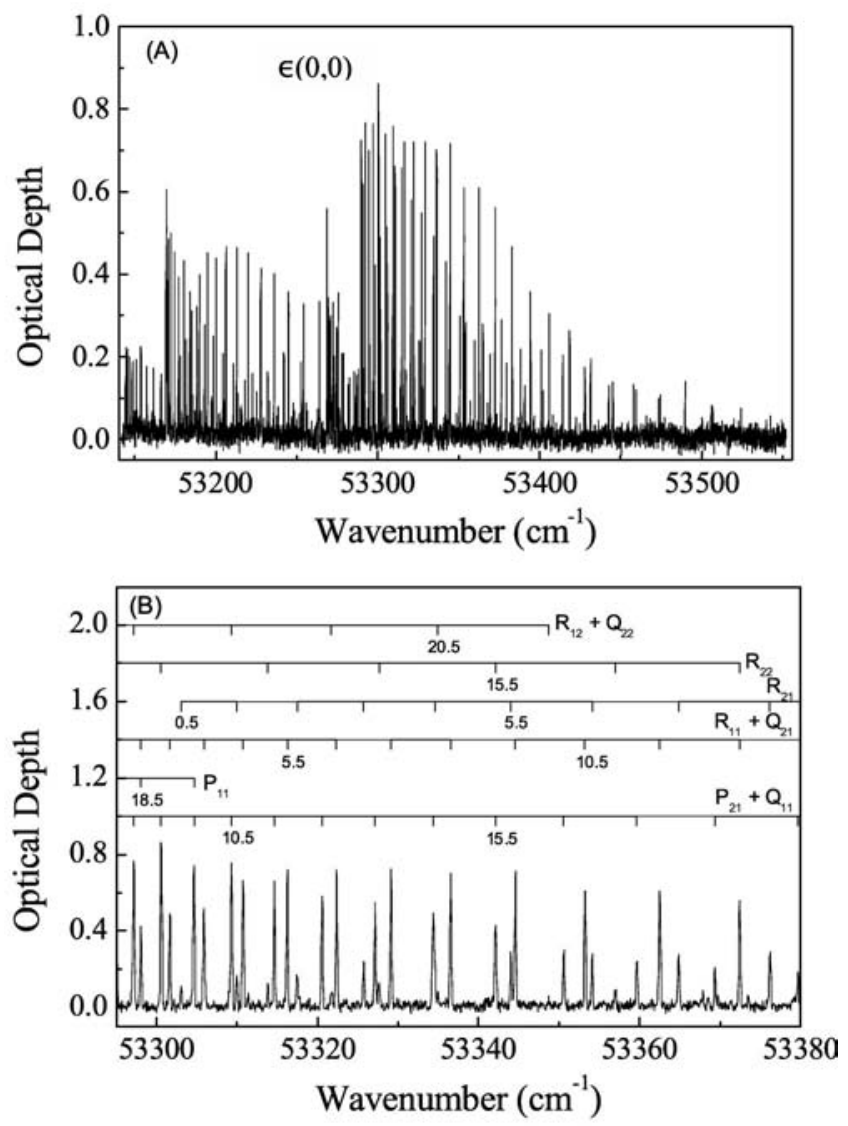

FIG. 1. The $\epsilon(0,0)$ band at 0.300 Torr of $\mathrm{NO}$ with $4.50 \mathrm{~cm}$ path length is shown in (A). An expanded portion of the spectrum with the transition assignments is displayed in (B).

\section{RESULTS AND DISCUSSION}

The transmission spectra were converted to optical depth by taking the logarithms of the intensity and fitting a smooth continuum to the regions between the lines. The absorption lines were fitted to Voigt profiles using the spectral reduction routine GREMLIN. ${ }^{25}$ Line parameters such as line position, linewidth, and integrated intensity of individual lines were determined through a nonlinear least-squares iterative procedure. The Voigt profile for the NO lines should be a convolution of Gaussian due to the Doppler broadening and a Lorentzian arising from predissociation. In this case the Gaussian component of the best-fit Voigt function had a full width at half maximum (FWHM) of $0.175 \mathrm{~cm}^{-1}$ which is significantly larger than the value of $0.12 \mathrm{~cm}^{-1}$ expected for the Doppler width in our experimental conditions. This anomalous Gaussian width is considered to be due to drifts in alignment causing very small wave number shifts over the long observation periods. ${ }^{26}$

\section{A. Line positions and molecular constants}

The absorption spectrum of $\mathrm{NO}$ in the wavelength region $186.7-188.2 \mathrm{~nm}$ consists of the $\gamma(4,0), \beta(8,0)$, and $\epsilon(0,0)$ bands. The strong $\epsilon(0,0)$ band dominated the observed spectral region and the $\gamma(4,0)$ and $\beta(8,0)$ bands are weak. The $\epsilon(0,0)$ band is shown in Fig. 1(A), and a portion of the spectrum is expanded in Fig. 1(B) to show the detail of the rota- 
TABLE I. Observed wave numbers of the $\epsilon(0,0)$ band of NO. ${ }^{a}$

\begin{tabular}{|c|c|c|c|c|c|c|c|c|}
\hline$J$ & $P_{11}$ & $R_{11}+Q_{21}^{\mathrm{b}}$ & $P_{21}+Q_{11}^{\mathrm{b}}$ & $R_{21}$ & $P_{22}+Q_{12}^{\mathrm{b}}$ & $R_{22}$ & $P_{12}$ & $R_{12}+Q_{22}{ }^{\mathrm{b}}$ \\
\hline 0.5 & & 53295.091 & & 53303.054 & & & & \\
\hline 1.5 & 53286.099 & $53298.041 \mathrm{~B}$ & $53291.084 b$ & 53309.957 & $53170.148 b$ & $53189.811 \mathrm{~B}$ & 53166.192 & 53178.147 \\
\hline 2.5 & 53281.722 & 53301.637 & $53289.660 \mathrm{~b}$ & 53317.489 & $53169.490 \mathrm{~B}$ & 53197.369 & 53161.544 & 53181.492 \\
\hline 3.5 & 53277.988 & 53305.852 & $53289.932 b$ & 53325.742 & 53169.490B & 53205.288 & 53157.514 & 53185.344 \\
\hline 4.5 & $53274.904 \mathrm{~b}$ & 53310.731 & $53290.752 b$ & $53334.580 \mathrm{~b}$ & $53169.894 \mathrm{~b}$ & 53213.713 & 53153.944 & 53189.811B \\
\hline 5.5 & $53272.397 \mathrm{~b}$ & 53316.227 & $53292.255 \mathrm{~B}$ & $53344.040 \mathrm{~b}$ & 53170.877 & 53222.657 & 53150.958 & 53194.783 \\
\hline 6.5 & $53270.586 b$ & 53322.360 & 53294.407 & 53354.135 & 53172.415 & 53232.135 & 53148.529 & 53200.292 \\
\hline 7.5 & $53269.435 b$ & 53329.131 & $53297.169 \mathrm{~B}$ & 53364.873 & 53174.493 & $53242.189 \mathrm{~B}$ & 53146.637 & 53206.355 \\
\hline 8.5 & $53268.890 \mathrm{~B}$ & 53336.531 & $53300.578 \mathrm{~B}$ & 53376.227 & 53177.115 & 53252.764 & $53145.286 \mathrm{~B}$ & 53212.954 \\
\hline 9.5 & $53268.890 \mathrm{~B}$ & $53344.565 b$ & $53304.639 \mathrm{~B}$ & 53388.255 & 53180.285 & $53263.972 \mathrm{~B}$ & $53144.413 \mathrm{~B}$ & 53220.092 \\
\hline 10.5 & $53269.664 \mathrm{~b}$ & 53353.226 & $53309.310 \mathrm{~B}$ & 53400.886 & 53183.999 & $53275.556 \mathrm{~B}$ & $53144.413 \mathrm{~B}$ & 53227.789 \\
\hline 11.5 & $53270.997 \mathrm{~b}$ & 53362.517 & 53314.633 & 53414.181B & 53188.258 & 53287.758 & 53144.413B & 53236.037 \\
\hline 12.5 & $53272.974 \mathrm{~b}$ & $53372.449 \mathrm{~B}$ & 53320.574 & 53428.019 & 53193.070 & $53300.578 \mathrm{~B}$ & $53145.286 \mathrm{~B}$ & 53244.824 \\
\hline 13.5 & 53275.556B & 53382.982 & $53327.140 \mathrm{~b}$ & 53442.516 & 53198.440 & 53313.802 & & 53254.157 \\
\hline 14.5 & 53278.807 & 53394.154 & $53334.348 b$ & 53457.657 & 53204.350 & $53327.644 \mathrm{~b}$ & & $53263.972 B$ \\
\hline 15.5 & 53282.643 & 53405.956 & $53342.129 \mathrm{~B}$ & 53473.415 & 53210.829 & $53342.129 \mathrm{~B}$ & & $53274.476 \mathrm{~b}$ \\
\hline 16.5 & 53287.154 & 53418.373 & 53350.595 & 53489.759B & 53217.843 & 53356.982 & & 53285.465 \\
\hline 17.5 & 53292.255B & 53431.409 & 53359.670 & & 53225.407 & 53372.449B & & 53297.169B \\
\hline 18.5 & 53298.041B & 53445.057 & 53369.363 & & 53233.527 & & & $53309.310 \mathrm{~B}$ \\
\hline 19.5 & 53304.639B & 53459.339 & 53379.658 & & $53242.189 \mathrm{~B}$ & & & 53321.724 \\
\hline 20.5 & & 53474.268 & 53390.615 & & & & & $53334.942 \mathrm{~b}$ \\
\hline 21.5 & & $53489.759 \mathrm{~B}$ & 53402.095 & & & & & 53348.692 \\
\hline 22.5 & & & 53414.181B & & & & & \\
\hline
\end{tabular}

${ }^{a}$ The uncertainty in the absolute values is $0.1 \mathrm{~cm}^{-1}$, but the precision of the relative values is better than $0.01 \mathrm{~cm}^{-1}$ for all except the very weak lines. Blended lines followed by B are observed as a single line and by $\mathrm{b}$ are observed as an incompletely resolved complex.

${ }^{\mathrm{b}}$ Lines of the two branches are overlapped.

tional structure. Due to a very small spin rotation constant $\gamma$ in the $D^{2} \Sigma^{+}$state, the separations between the $F_{1}$ and $F_{2}$ components are small. Among the 12 branches expected from a ${ }^{2} \Sigma^{+}-{ }^{2} \Pi$ transition, only eight branches were identified, of which four are the unresolved $Q_{11}+P_{21}, R_{11}$ $+Q_{21}, P_{22}+Q_{12}$, and $R_{12}+Q_{22}$ branches and the other four are the $P_{11}, R_{22}, R_{21}$, and $P_{21}$ branches. The spectral lines were fitted well using a Voigt profile with the Doppler width of $0.175 \mathrm{~cm}^{-1}$ and yielded line width with a FWHM about $0.20-0.22 \mathrm{~cm}^{-1}$. The measured line positions of the $\epsilon(0,0)$ band are listed in Table I, where partly resolved complexes and entirely blended lines are labeled with $\mathrm{b}$ and $\mathrm{B}$, respectively. As stated in Sec. II, the uncertainty in the absolute wave number is $0.1 \mathrm{~cm}^{-1}$, while for the strongest lines the relative uncertainty is $0.01 \mathrm{~cm}^{-1}$. Differences in the measured line positions between our present results and the unpublished data of Miescher ${ }^{27}$ are plotted in Fig. 2. The average shift of $+0.029 \pm 0.079 \mathrm{~cm}^{-1}$ is entirely consistent with the claimed uncertainties of Miescher's measurements of $0.1 \mathrm{~cm}^{-1}$. In addition, about 20 lines belonging to the $\beta(8,0)$ band were also identified in regions between transition lines of the $\epsilon(0,0)$ band. However, these $\beta(8,0)$ band lines are very weak and were not included for further processing.

The rotational term values of the $v=0$ level of the $D^{2} \Sigma^{+}$state [upper state of the $\epsilon(0,0)$ band] were obtained by adding the term values of the $v=0$ level of the $X^{2} \Pi$ state to the wave numbers of the observed transition lines. Accurate rotational term values of the $X^{2} \Pi$ state reported by Amiot $e t a l .{ }^{28}$ by using high-resolution FT infrared spectroscopy were used, for which the $\Omega=1 / 2, J=0.5$, and $e$ level was taken as the relative zero. The upper-state term values obtained from various branches were averaged, and the re- sults are listed in Table II. Braun et al. ${ }^{17}$ obtained the term values of the $D(0)$ level by a combination of their measurements of the $D \rightarrow C$ band and $C \leftarrow X$ band by Murray et al. ${ }^{18}$ Differences between ours and their values are included in Table II, and also plotted in Fig. 3, where open circles and triangles are presented for $F_{1 e}$ and $F_{2 f}$, respectively. The averaged difference for $F_{1 e}$ levels is $0.083 \pm 0.013 \mathrm{~cm}^{-1}$, and the difference of the first 14 levels of $F_{2 f}$ is 0.120 $\pm 0.010 \mathrm{~cm}^{-1}$. The average shifts are presented as dashed lines in Fig. 3. The higher $J$ levels of $F_{2 f}$ are shifted away from the average. It is not at all clear at this point as to what was the reason for the discrepancy. The weak perturbation

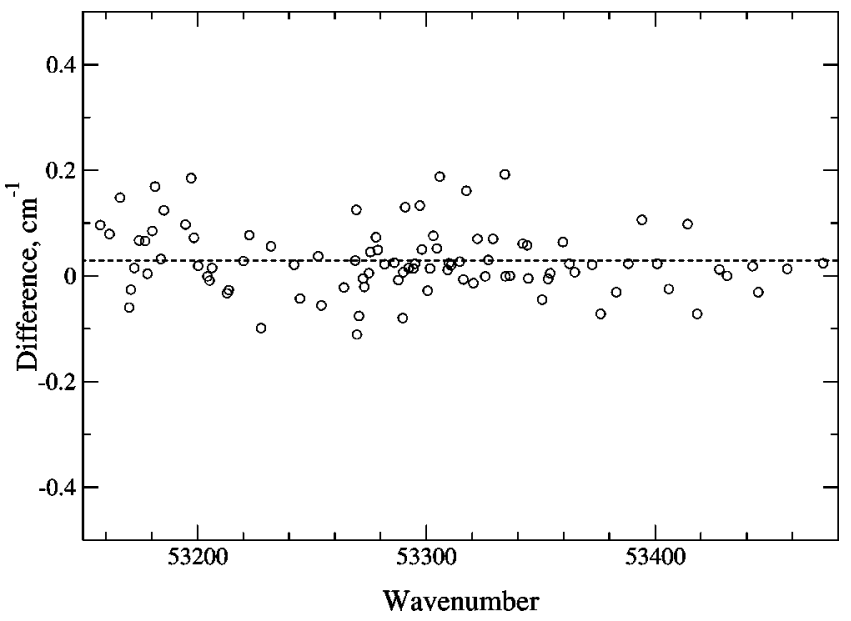

FIG. 2. Differences in the observed line positions between our results and Miescher (Ref. 27). The differences are given by the open circles whereas the dotted horizontal line represents the average shift of $+0.029 \mathrm{~cm}^{-1}$. 
TABLE II. The term values of the $D(0){ }^{2} \Sigma^{+}$levels of $\mathrm{NO}^{\mathrm{a}}\left(\mathrm{cm}^{-1}\right)$.

\begin{tabular}{|c|c|c|c|c|}
\hline \multirow[b]{2}{*}{$J$} & \multirow{2}{*}{$\begin{array}{c}F_{1}(J) \\
e\end{array}$} & \multirow{2}{*}{$\begin{array}{c}F_{2}(J) \\
\quad f\end{array}$} & \multicolumn{2}{|c|}{ Diffrences $\mathrm{BHV}^{\mathrm{b}}$-present } \\
\hline & & & $e$ & $f$ \\
\hline 0.5 & $53291.107(02)$ & $53295.062(00)$ & 0.102 & 0.135 \\
\hline 1.5 & $53295.074(16)$ & $53303.059(06)$ & 0.114 & 0.108 \\
\hline 2.5 & $53303.058(08)$ & $53315.017(24)$ & 0.088 & 0.097 \\
\hline 3.5 & $53315.013(26)$ & $53330.904(17)$ & 0.076 & 0.134 \\
\hline 4.5 & $53330.907(14)$ & $53350.835(13)$ & 0.108 & 0.114 \\
\hline 5.5 & $53350.825(14)$ & $53374.726(16)$ & 0.094 & 0.113 \\
\hline 6.5 & $53374.719(09)$ & $53402.584(18)$ & 0.082 & 0.121 \\
\hline 7.5 & $53402.581(16)$ & $53434.430(08)$ & 0.081 & 0.119 \\
\hline 8.5 & $53434.427(10)$ & $53470.248(19)$ & 0.074 & 0.121 \\
\hline 9.5 & $53470.238(12)$ & $53510.037(20)$ & 0.079 & 0.128 \\
\hline 10.5 & $53510.026(15)$ & $53553.806(21)$ & 0.083 & 0.130 \\
\hline 11.5 & $53553.795(13)$ & $53601.555(27)$ & 0.078 & 0.121 \\
\hline 12.5 & $53601.530(26)$ & $53653.270(20)$ & 0.081 & 0.119 \\
\hline 13.5 & $53653.246(24)$ & $53708.938(23)$ & 0.072 & 0.131 \\
\hline 14.5 & $53708.917(33)$ & $53768.574(16)$ & 0.076 & 0.143 \\
\hline 15.5 & $53768.574(16)$ & $53832.197(30)$ & 0.063 & 0.133 \\
\hline 16.5 & $53832.170(37)$ & $53899.758(36)$ & 0.074 & 0.145 \\
\hline 17.5 & $53899.740(13)$ & $53971.289(31)$ & 0.073 & 0.189 \\
\hline 18.5 & $53971.268(12)$ & $54046.753(31)$ & 0.075 & 0.175 \\
\hline 19.5 & $54046.739(15)$ & $54126.194(08)$ & 0.090 & 0.179 \\
\hline 20.5 & $54126.194(08)$ & $54209.570(84)$ & 0.078 & 0.204 \\
\hline 21.5 & $54209.570(84)$ & $54296.86(13)$ & 0.095 & 0.26 \\
\hline 22.5 & $54296.86(13)$ & & & \\
\hline
\end{tabular}

The uncertainties shown are the standard deviations and refer to relative values. The uncertainty in the absolute values is $0.1 \mathrm{~cm}^{-1}$ (see text).

${ }^{\mathrm{b}}$ BHV: Braun et al. (Ref. 17).

observed at $F_{2}$ (3.5) by Barrow and Miescher ${ }^{15}$ results in the extra level as reported by Braun et al. ${ }^{17}$ but we have not identified any extra lines belonging to this level.

Since the spin components of the $D^{2} \Sigma^{+}$state are not resolved, the term values were fitted to the expression

$$
T V=T_{0}+B J(J+1)-D J^{2}(J+1)^{2},
$$

where $T_{0}$ is the band origin and $B$ and $D$ are the rotational parameters. Our determined molecular constants and those from earlier workers are listed in Table III. The rms error of

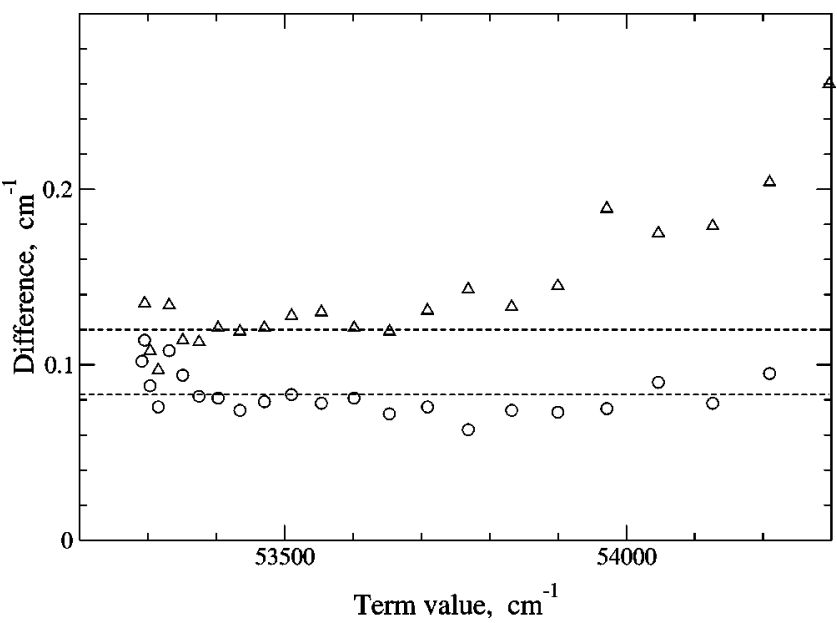

FIG. 3. Differences in the term values between our results and those of Braun $e t$ al. (Ref. 17). The differences are given by open circles for the $F_{1 e}$ and triangles for the $F_{2 f}$. The dashed lines represent the average shift of the $F_{1 e}$ and $F_{2 f}$.
TABLE III. The molecular constants of the $D(0)^{2} \Sigma^{+}$levels of NO $\left(\mathrm{cm}^{-1}\right)$.

\begin{tabular}{llll}
\hline \hline & \multicolumn{1}{c}{$T_{0}$} & \multicolumn{1}{c}{$B_{0}$} & $10^{6} D$ \\
\hline Present & $53291.10(1)^{\mathrm{a}}$ & $1.99107(5)$ & $6.6(1)$ \\
$\mathrm{AV}^{\mathrm{b}}$ & 53291.209 & & \\
$\mathrm{BM}^{\mathrm{c}}$ & 53291.2 & 1.9917 & \\
$\mathrm{OG}^{\mathrm{d}}$ & 53292.1 & 1.990 & 6 \\
$\mathrm{GY}^{\mathrm{e}}$ & 53292.6 & 1.992 & 6.1 \\
$\mathrm{GSV}^{\mathrm{f}}$ & 53291.9 & 1.9917 & \\
\hline
\end{tabular}

${ }^{a}$ The uncertainty in $T_{0}$ is dominated by the $0.1 \mathrm{~cm}^{-1}$ uncertainty in the absolute wave number.

${ }^{\mathrm{b}} \mathrm{AV}$ : Amiot and Verges (Ref. 16).

${ }^{\mathrm{c}} \mathrm{BM}$ : Barrow and Miescher (Ref. 15).

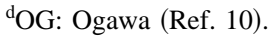

${ }^{\mathrm{e}} \mathrm{GY}$ : Gaydon (Ref. 13).

${ }^{\mathrm{f}}$ GSV: Gerö, Schmid, and Von Szily (Ref. 12).

our fit was $0.013 \mathrm{~cm}^{-1}$. The agreement with earlier work is excellent, but we believe our uncertainties to be smaller because of the high resolution and wave number accuracy of the observations.

\section{B. Integrated cross sections and oscillator strengths}

The fitting procedure employed under GREMLIN (Ref. 25) also evaluates integrated areas for the lines fitted. These are unaffected by the anomalous widths of the lines, provided that the residuals of the fit are comparable with the noise levels in the observed spectrum. This was found to be the case when the Gaussian contribution of the Voigt profile was held constant at $0.175 \mathrm{~cm}^{-1}$. The integrated cross sections derived from the areas and the total integrated cross sections of NO are given in Table IV. The integrated cross sections of blended lines have been separated by using branching ratios observed for other transitions together with the Boltzmann population distribution. The values listed in Table IV can be divided by the fractional populations of the rotational levels to obtain values proportional to the line oscillator strengths.

The uncertainties in the integrated cross sections arise from noise and the errors in the measurement of path length and pressure. The relative importance of these components in the uncertainty can be evaluated by making measurements at different sample gas pressures and path lengths, as was done for a band previously analyzed. ${ }^{2}$ These measurements showed that noise made the dominant contribution to the uncertainty and that, because photon noise is evenly distributed throughout an FT spectrum, the typical uncertainty for a single point in a cross section was $\pm 2.7 \times 10^{-18} \mathrm{~cm}^{2}$ for all points on the linear portion of the curve of growth. For the unblended strong lines of integrated cross sections 2.3 $\times 10^{-17} \mathrm{~cm}^{2} \mathrm{~cm}^{-1}$, the uncertainty due to noise is estimated to be $3 \%-4 \%$. For the weaker lines $1 \times 10^{-17} \mathrm{~cm}^{2} \mathrm{~cm}^{-1}$, the uncertainty is about $10 \%$. The large uncertainties of weaker lines would not significantly affect the integrated cross sections of the band, because the strong lines determine the integrated cross sections predominantly. The total integrated cross section for the band is estimated to be about $5 \%$.

The band oscillator strength of a $\left(v^{\prime}, v^{\prime \prime}\right)$ band is given by 
TABLE IV. Integrated cross sections of lines of the $\epsilon(0,0)$ band of NO in units of $10^{-18} \mathrm{~cm}^{2} \mathrm{~cm}^{-1}$.

\begin{tabular}{|c|c|c|c|c|c|c|c|c|}
\hline$J$ & $P_{11}$ & $R_{11}+Q_{21}^{\mathrm{a}}$ & $P_{21}+Q_{11}^{\mathrm{a}}$ & $R_{21}$ & $P_{22}+Q_{12}^{\mathrm{a}}$ & $R_{22}$ & $P_{12}$ & $R_{12}+Q_{22}{ }^{\mathrm{a}}$ \\
\hline 0.5 & & 10.22 & & 4.29 & & & & \\
\hline 1.5 & 3.63 & 15.56 & 6.10 & 6.04 & 15.28 & 0.96 & 9.52 & 8.75 \\
\hline 2.5 & 6.85 & 21.68 & 29.80 & 9.47 & 14.86 & 7.38 & 10.52 & 12.69 \\
\hline 3.5 & 9.05 & 28.24 & 34.76 & 10.14 & 19.01 & 4.55 & 8.56 & 21.38 \\
\hline 4.5 & 12.92 & 29.40 & 27.81 & 9.23 & 15.14 & 6.69 & 16.14 & 20.78 \\
\hline 5.5 & 16.32 & 29.89 & 31.12 & 11.50 & 23.43 & 9.77 & 14.12 & 21.88 \\
\hline 6.5 & 13.83 & 33.58 & 31.26 & 12.48 & 22.57 & 9.98 & 13.06 & 21.82 \\
\hline 7.5 & 15.67 & 32.98 & 38.60 & 13.13 & 20.35 & 8.06 & 11.05 & 22.14 \\
\hline 8.5 & 14.80 & 34.47 & 36.10 & 15.61 & 20.83 & 8.53 & 7.71 & 21.96 \\
\hline 9.5 & 12.11 & 33.05 & 33.01 & 13.47 & 20.72 & 8.42 & 8.74 & 22.96 \\
\hline 10.5 & 9.50 & 29.54 & 30.81 & 8.90 & 18.95 & 6.69 & 5.98 & 26.28 \\
\hline 11.5 & 10.15 & 29.10 & 28.68 & 9.49 & 16.65 & 5.71 & 4.74 & 19.10 \\
\hline 12.5 & 9.16 & 25.04 & 26.15 & 8.69 & 14.49 & 4.70 & 2.65 & 15.71 \\
\hline 13.5 & 9.58 & 23.49 & 24.28 & 8.32 & 12.81 & 4.42 & & 14.03 \\
\hline 14.5 & 8.75 & 18.91 & 23.42 & 6.10 & 9.36 & 6.26 & & 14.66 \\
\hline 15.5 & 6.39 & 15.79 & 18.89 & 4.13 & 8.55 & 4.25 & & 10.87 \\
\hline 16.5 & 5.86 & 12.86 & 15.32 & 1.99 & 7.84 & 5.22 & & 8.03 \\
\hline 17.5 & 3.05 & 10.98 & 12.72 & & 9.09 & 1.45 & & 4.24 \\
\hline 18.5 & 2.63 & 10.21 & 9.15 & & 4.47 & & & 5.30 \\
\hline 19.5 & 1.84 & 8.11 & 9.55 & & 3.92 & & & 4.71 \\
\hline 20.5 & & 7.11 & 6.81 & & & & & 3.04 \\
\hline 21.5 & & 3.49 & 7.12 & & & & & 2.16 \\
\hline 22.5 & & & 5.42 & & & & & \\
\hline Total $^{\text {b }}$ & 172.09 & 463.70 & 486.88 & 152.98 & 278.32 & 103.04 & 112.79 & 302.49 \\
\hline Extended $^{c}$ & 180.37 & 482.01 & 512.18 & 165.26 & 293.51 & 111.75 & 119.79 & 318.62 \\
\hline
\end{tabular}

${ }^{a}$ Lines of the two branches are overlapped.

${ }^{\mathrm{b}}$ Total integrated cross sections of the band: $2072.29 \times 10^{-18}$

${ }^{\mathrm{c}}$ Extended total of the band: $2183.49 \times 10^{-18}$.

$$
f\left(v^{\prime}, v^{\prime \prime}\right)=\frac{m c^{2}}{\pi e^{2}} \frac{1}{\widetilde{N}\left(v^{\prime \prime}\right)} \int \sigma(\nu) d \nu
$$

in which $\widetilde{N}\left(v^{\prime \prime}\right)$ is the fractional Boltzmann population of the absorbing vibrational level-in this case, since $v^{\prime \prime}=0$, this quantity is unity - and the integration of the cross section $\sigma(\nu)$ is performed over all of the rotational lines belonging to the $\epsilon(0,0)$ band. The total integrated cross sections of observed lines for each branch are presented in Table IV as "Total." Observations of the rotational lines are mostly limited to $J \leqslant 22.5$. The effects from higher- $J$ lines cannot be ignored. The contribution of the higher- $J$ lines was obtained by extending the total integrated cross section of the lines up to $J=36.5$ as expected from the Boltzmann distribution. Figure 4 is a plot of the total integrated cross sections of the $P_{11}$ branch of the $\epsilon(0,0)$ band versus $J$. The solid curve in the figure was obtained by fitting those experimental points and the extended contribution was estimated by extrapolating the curve to $J=36.5$. The results of the extended contribution are also listed in Table IV as "Extended."

The band oscillator strength determined for the $\epsilon(0,0)$ band and other bands measured earlier are given in Table V, where numbers in bold are values for the single band. The uncertainty in our measured band oscillator strength is estimated to be about $5 \%$, which includes uncertainties from line intensity measurements and extrapolation. Our value of the $\epsilon(0,0)$ band is very close to all other measurements except that of Lugue and $\mathrm{Crosley}^{24}$ and Chan et al. ${ }^{23}$ The $\epsilon(0,0)$ band is blended with the weaker $\beta(8,0)$ and $\gamma(0,0)$ bands, and ours are the only experimental measurements that allow these band oscillator strength to be treated separately. Since the $\beta, \epsilon, \gamma$, and $\delta$ systems of NO are overlapping in the same spectral region and the band oscillator strength of vibronic bands of these systems are interrelated, we would like to postpone our discussion of the band oscillator strength of various NO band systems in the wavelength region 160-195 $\mathrm{nm}$ until our analysis of bands in this region is completed.

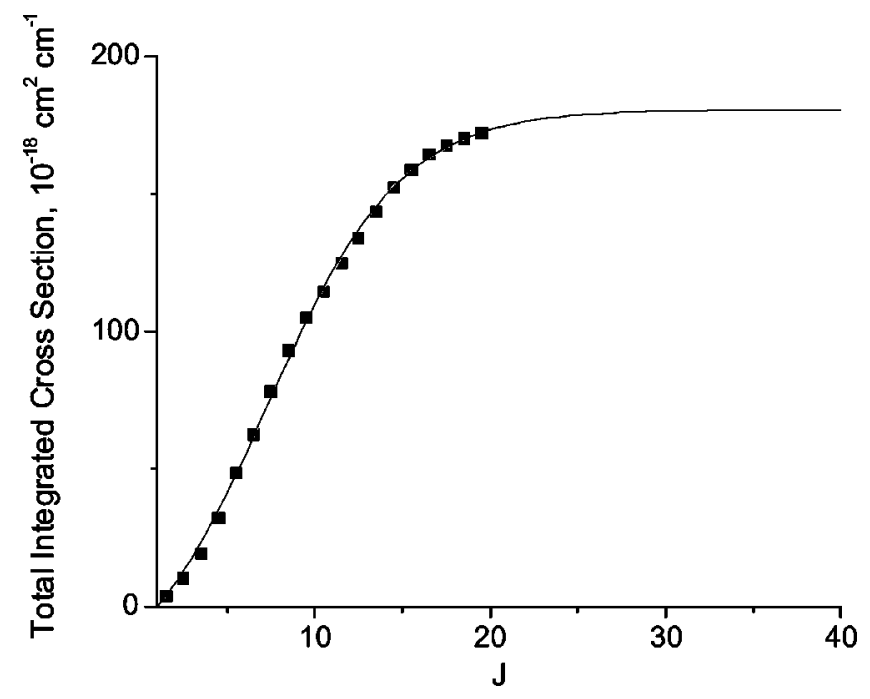

FIG. 4. Line-by-line total integrated cross sections of the $P_{11}$ branch of the $\epsilon(0,0)$ band. 
TABLE V. Band oscillator strength $\left(10^{-3}\right)$ of NO. ${ }^{\text {a }}$

\begin{tabular}{|c|c|c|c|c|c|c|c|}
\hline Main band & $\gamma(3,0)$ & $\beta(6,0)$ & $\epsilon(0,0)$ & $\beta(9,0)$ & $\delta(1,0)$ & $\epsilon(1,0)$ & $\beta(11,0)$ \\
\hline FTS/VUV & 0.27 & 0.048 & 2.47 & 0.27 & 5.4 & 2.9 & 0.34 \\
\hline Luque and Crosley ${ }^{\mathrm{c}}$ & 0.36 & & 1.87 & & & 3.7 & \\
\hline Chan et al. ${ }^{\mathrm{d}}$ & 0.36 & 0.037 & 2.75 & 0.31 & 6.0 & 4.6 & 0.65 \\
\hline Guest and Lee ${ }^{\mathrm{e}}$ & & & 2.51 & & 8.5 & 4.6 & \\
\hline Cieslik $^{\mathrm{f}}$ & 0.31 & & 2.53 & 0.36 & 5.6 & 6.5 & \\
\hline Callear and Pillingg & & & 2.54 & & & & \\
\hline Bethke $^{\mathrm{h}}$ & 0.36 & 0.046 & 2.54 & 0.36 & 5.8 & 4.6 & 0.36 \\
\hline \multirow[t]{2}{*}{ Blended band } & $\beta(6,0)$ & & $\beta(8,0)$ & & $\beta(10,0)$ & $\beta(11,0)$ & $\epsilon(1,0)$ \\
\hline & & & $\gamma(4,0)$ & & & $\gamma(5,0)$ & \\
\hline
\end{tabular}

${ }^{a}$ Values in bold are obtained for a single band.

bresent work for the $\epsilon(0,0)$ band and our published works (Refs. 1-5).

${ }^{c}$ Luque and Crosley (1999) (Ref. 24).

${ }^{\mathrm{d}}$ Chan, Cooper, and Brion (1993) (Ref. 23).

\section{ACKNOWLEDGMENTS}

This work was supported in part by NSF Division of Atmospheric Sciences Grant No. ATM-94-22854 to Harvard College Observatory and by the NASA Upper Atmospheric Research Program under Grant No. NAG5-484 to the Smithsonian Astrophysical Observatory. We also acknowledge the Paul Instrument Fund of the Royal Society for the development of the VUV-FT spectrometer. The FTS measurements at the Photon factory were made with the approval of the Photon Factory Advisory Committee (94G367). K.Y. thanks the Japan Society for the Promotion of Science for support. A.P.T. thanks NATO for its grant for international collaboration in research (Grant No. 890224).

${ }^{1}$ K. Yoshino, J. R. Esmond, W. H. Parkinson et al., J. Chem. Phys. 109, 1751 (1998).

${ }^{2}$ T. Imajo, K. Yoshino, J. R. Esmond et al., J. Chem. Phys. 112, 2251 (2000).

${ }^{3}$ J. Rufus, K. Yoshino, J. R. Esmond, A. P. Thorne, T. Imajo, K. Ito, and T. Matsui, J. Chem. Phys. 115, 3719 (2001).

${ }^{4}$ A. S.-C. Cheung, D. H.-Y. Lo, K. W.-S. Leung, K. Yoshino, A. P. Thorne, J. E. Murray, K. Ito, T. Matsui, and T. Imajo, J. Chem. Phys. 116, 155 (2002).

${ }^{5}$ J. Rufus, K. Yoshino, A. P. Thorne, J. E. Murray, T. Imajo, K. Ito, and T.

Matsui, J. Chem. Phys. 117, 10621 (2002).

${ }^{6}$ S. W. Leifson, Astrophys. J. 63, 73 (1926).
${ }^{\mathrm{e}}$ Guest and Lee (1981) (Ref. 22)

${ }^{\mathrm{f}}$ Cieslik (1977) (Ref. 20), only values from the curve of growth. ${ }^{\mathrm{g}}$ Callear and Pilling (1970) (Ref. 21).

${ }^{\text {h} B e t h k e ~(1959) ~(R e f . ~ 19) . ~}$

${ }^{7}$ E. Miescher and K. P. Huber, Spectroscopy, International Review of Science, Physical Chemistry Ser. 2, Vol. 3, edited by Ramsey (Butterworths, London, 1976).

${ }^{8}$ G. Herzberg and L. G. Mundie, J. Chem. Phys. 8, 263 (1940).

${ }^{9}$ Y. Tanaka, J. Sci. Res. Inst. (Tokyo) 43, 160 (1949).

${ }^{10}$ M. Ogawa, Sci. Light (Tokyo) 3, 90 (1955).

${ }^{11}$ G. Herzberg, A. Lagerqvist, and E. Miescher, Can. J. Phys. 34, 622 (1956).

${ }^{12}$ L. Gerö, R. Schmid, and F. K. Von Szily, Physica (Amsterdam) 11, 144 (1944).

${ }^{13}$ A. G. Gaydon, Proc. Phys. Soc. London 56, 95 (1944).

${ }^{14}$ A. G. Gaydon, Proc. Phys. Soc. London 56, 160 (1944).

${ }^{15}$ R. F. Barrow and E. Miescher, Proc. Phys. Soc., London, Sect. A 70, 219 (1957).

${ }^{16}$ C. Amiot and J. Verges, Phys. Scr. 25, 302 (1982).

${ }^{17}$ V. D. Braun, K. P. Huber, and M. Verloet, J. Mol. Spectrosc. 203, 65 (2000).

${ }^{18}$ J. E. Murray, K. Yoshino, J. R. Esmond, W. H. Parkinson, Y. Sun, A. Dalgarno, A. P. Thorne, and G. Cox, J. Chem. Phys. 101, 62 (1994).

${ }^{19}$ G. W. Bethke, J. Chem. Phys. 31, 662 (1959).

${ }^{20}$ S. Cieslik, Bull. Cl. Sci., Acad. R. Belg. 63, 884 (1977).

${ }^{21}$ A. B. Callear and M. J. Pilling, Trans. Faraday Soc. 66, 1886 (1970).

${ }^{22}$ J. A. Guest and L. C. Lee, J. Phys. B 14, 3401 (1981).

${ }^{23}$ W. F. Chan, G. Cooper, and C. E. Brion, Chem. Phys. 170, 111 (1993).

${ }^{24}$ J. Luque and D. R. Crosley, J. Phys. Chem. 111, 7405 (1999).

${ }^{25} \mathrm{~J}$. W. Brault (private communication).

${ }^{26}$ P. M. Dooley, B. R. Lewis, S. T. Gibson et al., J. Chem. Phys. 109, 3856 (1998).

${ }^{27}$ E. Miescher (private communication).

${ }^{28}$ C. Amiot, R. Basis, and G. Guelachvili, Can. J. Phys. 56, 251 (1978). 